\title{
Numerical results for the Edwards-Anderson spin-glass model at low temperature
}

\author{
Julio F. Fernández ${ }^{1,2, *}$ and Juan J. Alonso ${ }^{3}$ \\ ${ }^{1}$ Departamento de Física de la Materia Condensada, Universidad de Zaragoza, 50009 Zaragoza, Spain \\ ${ }^{2}$ Instituto Carlos I de Física Teórica y Computacional, Universidad de Granada, 18071 Granada, Spain \\ ${ }^{3}$ Física Aplicada I, Universidad de Málaga, 29071 Málaga, Spain
}

(Received 9 February 2013; published 25 April 2013)

\begin{abstract}
We have simulated Edwards-Anderson (EA) as well as Sherrington-Kirkpatrick systems of $L^{3}$ spins. After averaging over large sets of EA system samples of $3 \leqslant L \leqslant 10$, we obtain accurate numbers for distributions $p(q)$ of the overlap parameter $q$ at very low-temperature $T$. We find $p(0) / T \rightarrow 0.233(4)$ as $T \rightarrow 0$. This is in contrast with the droplet scenario of spin glasses. We also study the number of mismatched links-between replica pairs - that come with large scale excitations. Contributions from small scale excitations are discarded. We thus obtain for the fractal dimension of outer surfaces of $q \sim 0$ excitations in the EA model $d_{s} \rightarrow 2.59(3)$ as $T \rightarrow 0$. This is in contrast with $d_{s} \rightarrow 3$ as $T \rightarrow 0$ that is predicted by mean-field theory for the macroscopic limit.
\end{abstract}

\section{INTRODUCTION}

Whether spin glasses are complex systems is an important issue. We have discussed this in some detail in Ref. 1, where we gave numerical evidence for fundamental differences between the spin-glass phases of the Edwards-Anderson ${ }^{2}$ (EA) and Sherrington-Kirkpatrick ${ }^{3}$ (SK) models. In short, we studied spikes in probability distributions, $p(q)$, of the overlap parameter, $q$, that vary widely over different sample systems. The variation of a suitably defined average spike width $w$ over the values of the linear system sizes $L$ we studied was shown to decrease sharply with $L$ in the SK model. Furthermore, rms deviations $\delta p$ away from $p(q)$ over different system samples (that is, over different realizations of quenched disorder) increase sharply with $L$. Such behavior is consistent with mean-field theory, which predicts the replica symmetry-breaking (RSB) scenario in which $w \rightarrow 0$ and $\delta p \rightarrow \infty$ in the macroscopic limit. ${ }^{4,5}$ Our results $^{1}$ for the EA model follow a different trend. Rather, $w$ and $\delta p$ become, within errors, independent of $L$ in the zero-temperature limit. (The statistics of spikes in overlap distributions in different system samples, which have been studied by Yucesoy ${ }^{6}$ et al., also point away from an RSB scenario, though this conclusion is criticized in Ref. 7.)

Much numerical work on the behavior of the EA model at low temperature stems from the observations of Moore ${ }^{8}$ et al., that Monte Carlo (MC) simulations had up to then been performed at temperatures that were too close to the critical temperature and therefore suffered from finite-size effects that could be misinterpreted as RSB behavior. Lowtemperature data for $p(q)$, which was the centerpiece of these considerations, were soon thereafter provided by Katzgraber, Palassini, and Young ${ }^{9}$ (KPY). A roughly constant value of $p(q \sim 0)$ over a $3 \leqslant L \leqslant 8$ size range was shown to be consistent with these data. This is, as in the RSB, not the droplet scenario ${ }^{10,11}$ of spin glasses. We did not report data for $p(q)$ in Ref. 1 , because they were essentially the same as KPY's, and our statistical errors did not decisively improve on them. We have since simulated sample sets which are over an order of magnitude larger than KPY's and cover a range of system sizes which is slightly larger. We are thus able to report here rather accurate data for temperatures as low as $0.16 T_{\mathrm{sg}}$, where $T_{\mathrm{sg}}$ is the spin-glass transition temperature.
In the so-called trivial-non-trivial (TNT) picture, proposed by Krzakala and Martin ${ }^{12}$ and by Palassini and Young ${ }^{13}$ (PY), $p(q)$ is size independent in the neighborhood of $q=0$, as in the RSB scenario, but the dimensionality $d_{s}$ of outer surfaces of $q \sim 0$ excitations is smaller than the dimensionality, $d$, of the space where spins are embedded. Values of $2.57 \lesssim$ $d_{s} \lesssim 2.62$ have been calculated by PY, KPY, and by Jörg and Katzgraber. ${ }^{14}$ However, Contucci et al. ${ }^{15}$ have obtained $d_{s}=3$ for the three-dimensional (3D) EA model. This would be in accordance with a RSB scenario. These two conflicting results were obtained by different methods. Fractions of mismatched links (FML) between replica pairs are calculated in both methods. All $2.57 \lesssim d_{s} \lesssim 2.62$ values ${ }^{9,13,14}$ were obtained (but see Ref. 16) from the rms deviation of the FML from its mean value (over time and system samples, as well as over all $q$ ). On the other hand, $d_{s}=3$ was obtained in Ref. 15 from the behavior of the mean FML, $f_{\mathrm{ml}}(q)$, for each observed value of $q$. More specifically, the $L \rightarrow \infty$ limit of $f_{\mathrm{ml}}(q \sim 0)$ was studied in Ref. 15 for $T \gtrsim 0.5 T_{\mathrm{sg}}$. This limit was argued to be nonzero, which is what one expects of a space fulfilling surface. This conclusion fits with the RSB scenario and clashes with the ones reached in Refs. 9, 13, and 14.

Most of this paper is devoted to the fractional number of link mismatches, $\overline{\Delta f_{\mathrm{ml}}} Q$ it costs to create an excitation with a $-Q<q<Q$ value. For a more precise definition of $\overline{\Delta f_{\mathrm{ml}}} Q$, consider first $\overline{f_{\mathrm{ml}}} Q$, which is the average FML given that $q$ is in a given $-Q<q<Q$ interval. Subtraction from $\overline{f_{\mathrm{ml}}} Q$ of the average FML given that $q$ is not in the $-Q<q<Q$ interval gives $\overline{\Delta f_{\mathrm{ml}}} Q$. Both $\overline{\Delta f_{\mathrm{ml}}} Q$ and ${\overline{f_{\mathrm{ml}}}}_{Q}$ have the same zero-temperature limit, but we believe $\frac{f_{\mathrm{ml}}}{\Delta f_{\mathrm{m}}} Q$ is the natural extension to nonzero temperatures of the FML of large size excitations in the ground state. Whereas $\overline{f_{\mathrm{ml}}} Q$ decreases as $T$ decreases, $\overline{\Delta f_{\mathrm{ml}}} Q$ increases. This enables us to bracket very low-temperature behavior and confidently make $T \rightarrow 0$ extrapolations. These notions stand out clearly in the frustrated box (FB) model which we define below.

The plan of this paper is as follows. In Sec. II, we define the models, the spin-overlap and link-overlap parameters, and the simulation procedure. In Sec. III, we report accurate data for $p(q)$ for EA and SK systems at very low temperature. These data show that, in the $3 \leqslant L \leqslant 10$ range, $p(q \sim 0)$ is independent of $L$ at very low temperatures. The conclusion 
$\mathrm{KPY}^{9}$ reached, that the EA model exhibits a clear trend away from the droplet scenario, is thus strengthened. In Sec. IV A we examine the large scale behavior of the FML in the FB model. This simple model helps to highlight the pitfalls that should be avoided in the interpretation of a nonzero macroscopic limit of FML. In Sec. IV B, we assign a (average) mismatching-link cost, $\overline{\Delta f_{\mathrm{ml}}} Q$, to an excitation with a $-Q<q<Q$ value. Numerical results for $\overline{\Delta f_{\mathrm{ml}}} Q$, which imply a fractal dimension of 2.59(3) for the surface associated to $\overline{\Delta f_{\mathrm{ml}}} Q$, are also given in Sec. IV B. Such a value of $d_{s}$, smaller than the 3D dimensionality of the space where spins are embedded, is in contradiction with mean-field theory predictions but is as envisioned in the TNT scenario ${ }^{12,13}$ of the EA model. We summarize our conclusions in Sec. V.

\section{MODELS, DEFINITIONS, AND PROCEDURE}

In all models we study, an Ising spin sits on each one of the $N \equiv L \times L \times L$ sites of a simple cubic lattice in three dimensions. We use periodic boundary conditions throughout. In the SK and EA models the interaction energy between a pair of spins at sites $i$ and $j$ is given by $J_{i j} \sigma_{i} \sigma_{j}$. We let $J_{i j}=$ $\pm 1 / \sqrt{N}$ randomly, without bias, for all $i j$ site pairs in the $\mathrm{SK}$ model. For the EA model, $J_{i j}=0$ unless $i j$ are nearestneighbor pairs, and we draw each nearest-neighbor bond $J_{i j}$ independently from unbiased Gaussian distributions of unit variance.

We let all temperatures be given in units of $1 / k_{B}$, where $k_{B}$ is Boltzmann's constant. Thus, the transition temperature $T_{\mathrm{sg}}$ between the paramagnetic and SG phase of the SK model is $T_{\mathrm{sg}}=1 .{ }^{3,5}$ For the EA model $T_{\mathrm{sg}} \simeq 0.95 .^{17}$

We let $\sigma_{i}^{(1)}$ stand for a spin at site $i$ of replica 1 of a given system, and similarly $\sigma_{i}^{(2)}$ for an identical replica, replica 2, of the same system. As usual, we define

$$
q \equiv N^{-1} \Sigma_{i} q_{i} \quad \text { and } \quad q_{i}=\sigma_{i}^{(1)} \sigma_{i}^{(2)},
$$

that is, $q$ is the average (over all sites) spin alignment between the states replicas 1 and 2 are in.

As in Refs. 12 and 13, we define the link overlap:

$$
q_{l} \equiv\left(N_{l}\right)^{-1} \Sigma_{\langle i j\rangle} \sigma_{i}^{(1)} \sigma_{j}^{(1)} \sigma_{i}^{(2)} \sigma_{j}^{(2)},
$$

where $N_{l}$ is the total number of links, and the sum is over all $i, j$ nearest-neighbor pairs (of which there are $3 N$ in the nearest-neighbor 3D EA model). The FML between replicas 1 and 2 is given by $f_{\mathrm{ml}}=\left(1-q_{l}\right) / 2$. In addition, as in Ref. 15, we define $f_{\mathcal{J} \text { ml }}(q)$ as the time average of the FML (for a sample with a given set $\mathcal{J}$ of bonds) which is observed over all time intervals while the value of the spin overlap is $q$. Unfortunately, the dimensionality $d_{s}$ of large scale excitations does not follow straightforwardly from the behavior of $f_{\mathcal{J} \mathrm{ml}}$. This is because smaller scale excitations contribute to $f_{\mathcal{J} \text { ml }}(q)$. More on this can be found in Sec. IV.

Let $\mathcal{F}(q)$ be any $q$ dependent function, such as $p(q)$ or $f_{\mathrm{ml}}(q)$. We define

$$
\overline{\mathcal{F}}^{Q} \equiv(2 Q)^{-1} \int_{-Q}^{Q} d q \mathcal{F}(q) .
$$

The advantage of working with $\overline{\mathcal{F}}^{Q}$ is that statistical errors for it are smaller than for $\mathcal{F}(q)$. Accordingly, most results below are given for $\bar{p}^{Q}$ and $\overline{f_{\mathrm{ml}}} Q$ rather than for $p(q)$ and $f_{\mathrm{ml}}(q)$. How statistical errors on $\bar{p}^{Q}$ depend on $Q$ is worked out in Appendix A.

In addition, we let $A_{\mathcal{J}}$ stand for the value of some observable $A$ on a sample defined by the set $\mathcal{J}$ of bonds, and we let $\left\langle A_{\mathcal{J}}\right\rangle_{\mathcal{J}}$ stand for the average over samples of $A_{\mathcal{J}}$. Thus, $p(q) \equiv\left\langle p_{\mathcal{J}}(q)\right\rangle_{\mathcal{J}}$ and $[\delta p(q)]^{2} \equiv\left\langle\left[p_{\mathcal{J}}(q)-p(q)\right]^{2}\right\rangle_{\mathcal{J}}$.

We make use of the parallel tempered MC method. ${ }^{18,19}$ Details on how we apply it to the EA and SK models are as specified in Ref. 1. However, some details differ. For all sizes of the EA model, temperatures are spaced here by 0.04 (0.08) in the $0.16 \leqslant T \leqslant 0.48(0.48 \leqslant T \leqslant 1.6)$ range. The rationale for this, as well as checks we perform in order to make sure equilibrium is reached, can be found in Appendix B. Temperatures of all SK systems were evenly spaced by $\Delta T=$ 0.04 in the whole $0.12 \leqslant T \leqslant 1.6$ range. For the FB model, $\Delta T=0.02(\Delta T=0.01)$ for all $4 \leqslant L \leqslant 8(L=12)$. Values for average swap success rates, $\alpha$, between pairs of EA and SK systems at the lowest two temperatures are given in Table I. Larger values of $\alpha$ are observed for higher temperatures. In the FB model, the smallest value of $\alpha$, which we give in Table I, is observed in the critical region.

The number, $N_{s}$, of sample systems we average over is as specified in Table I, much larger here than in Ref. 1. We have tried not to make $N_{s}$ smaller with increasing $L$. This is because, as we show in Appendix A, statistical errors are independent of $L$, because of non-self-averaging. (For $L=10$, we could only do 20000 samples. That took some 50 years worth of computer time.)

\section{AVERAGE $q$ DISTRIBUTIONS AT LOW TEMPERATURES}

Plots of $p(q)$ vs $q$ are shown in Fig. 1 for EA systems of various sizes at $T=0.16$. Plots of $\bar{p}^{Q} / T$ vs $T$ are shown in Fig. 2 for $Q=1 / 4$ and $1 / 2$. Error sizes are clearly smaller

TABLE I. Number of samples, $N_{s}$, and the number of MC sweeps $\tau_{s}$ taken for equilibration as well as for subsequent averaging. The

\begin{tabular}{|c|c|c|c|c|c|c|c|c|c|}
\hline \multirow[b]{2}{*}{$L$} & \multicolumn{3}{|c|}{ SK } & \multicolumn{5}{|c|}{ EA } & \multirow{2}{*}{$\frac{\mathrm{FB}}{4-12}$} \\
\hline & 4 & 6 & 8 & 4 & 5 & 6 & 8 & 10 & \\
\hline$\tau_{s}$ & $10^{5}$ & $10^{5}$ & $10^{5}$ & $10^{4}$ & $10^{4}$ & $10^{5}$ & $10^{6}$ & $10^{7}$ & $10^{6}$ \\
\hline$N_{s}$ & $10^{5}$ & $10^{5}$ & $10^{5}$ & $10^{5}$ & $10^{5}$ & $10^{5}$ & $10^{5}$ & $2 \times 10^{4}$ & 1 \\
\hline$\alpha$ & 0.7 & 0.5 & 0.4 & 0.7 & 0.6 & 0.5 & 0.5 & 0.45 & $>0.3$ \\
\hline
\end{tabular}
average over samples of the swap success rate, $\alpha$, between EA and SK systems at their two lowest temperatures, and between systems in the critical region of the FB system, are also given. (Other swap rates are larger.) 


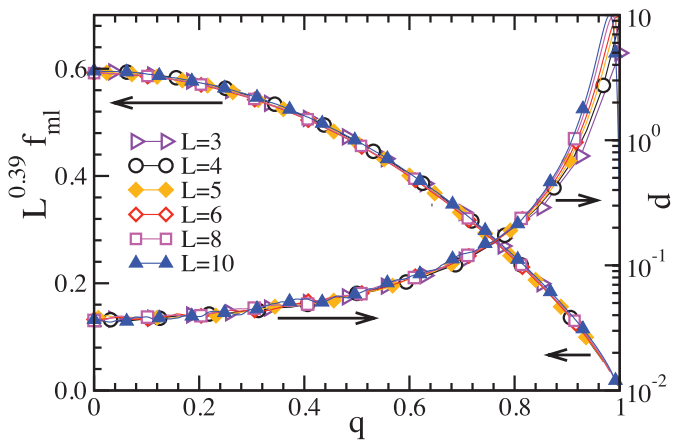

FIG. 1. (Color online) Plots of $L^{0.39} f_{\mathrm{ml}}$ and of $p$ vs $q$, for EA systems of $L^{3}$ spins, for the values of $L$ shown, at $T=0.16$. For clarity, only a fraction of all data points are shown, but the lines shown go through every one of them. The normalization condition $\int_{-1}^{1} d q p(q)=1$ is satisfied.

for the larger value of $Q$. Simulation details, such as sample numbers and running times, are given in Table I.

For comparison, plots of $\bar{p}^{Q} / T$ vs $T$ for SK systems of various sizes are shown in Fig. 3 for $Q=1 / 2$ and $1 / 4$. We note that $\bar{p}^{Q} \sim T$ if $T \lesssim 0.3 T$, independently of $L$, following mean-field predictions, ${ }^{5,20}$ for the SK model.

For a more accurate picture of how $\bar{p}^{Q}$ varies with $L$ in the EA model, we show $\log -\log$ plots of $\bar{p}^{Q} / T$ vs $L$, for $Q=1 / 4$ and $T=0.2$ in Fig. 4. For comparison, we also show data points from the KPY paper for the same temperature and $Q=0.2^{21}$

The best fit of $\bar{p}^{Q} \propto L^{-\theta}$ to the data points shown in Fig. 4 gives $\theta \simeq 0.008$. Fits following from letting $\theta=0.04$ and -0.02 give $\chi^{2}$ parameters that are over twice as large as the one for $\theta=0.008$. (See the figure legend for further details.) For higher temperatures, up to $T \simeq 0.4$, as well as for $Q=1 / 2$ and all $T \lesssim 0.5$, all error bars are smaller than the ones shown in Fig. 4, and all best fits of $\bar{p}^{Q} \propto L^{-\theta}$ to the data give $|\theta|<$ 0.01 . Thus, future generation of more accurate data that would give $\theta>0.04$ for the $3 \leqslant L \leqslant 10$ range is rather unlikely.

Finally, $Q \rightarrow 0$ and $T \rightarrow 0$ extrapolations give $p(0) / T \rightarrow$ $0.51(3)$ for the SK model. Similarly, $p(0) / T \rightarrow 0.233(4)$ as $T \rightarrow 0$ follows from the plots shown in Fig. 2 for the EA model.

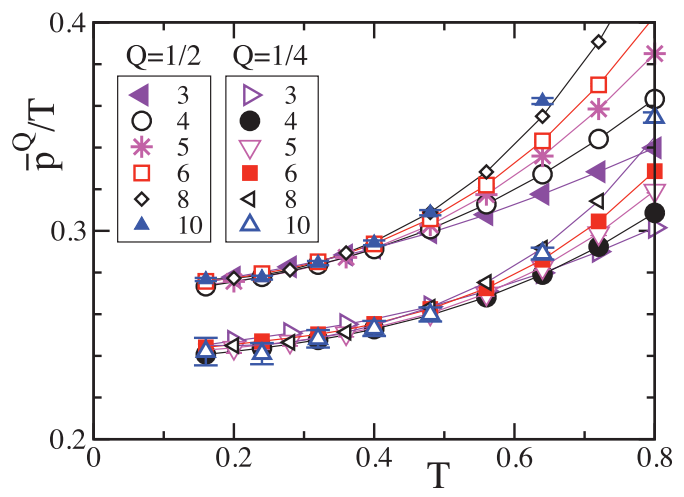

FIG. 2. (Color online) Plots of $\bar{p}^{Q}$ vs $T$ for EA systems of $L \times$ $L \times L$ spins, for the values of $L$ shown, and $Q=1 / 4$ and $1 / 2$. Icons for all $L \leqslant 8$ cover their error bars. For better visibility of data points, not all of them are shown, but lines go through every one of them.

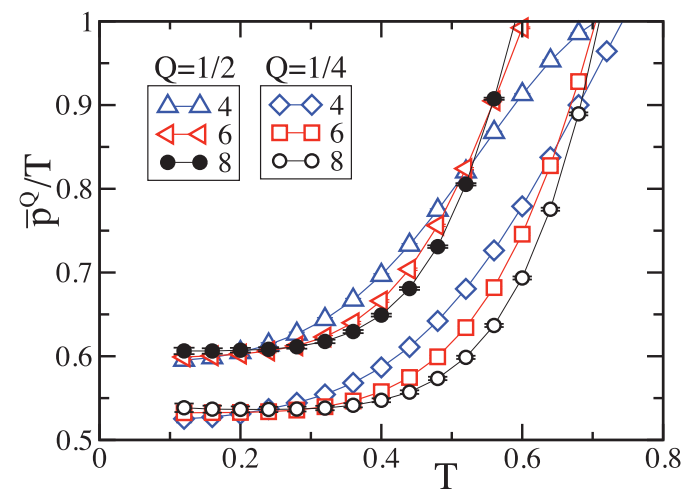

FIG. 3. (Color online) (a) Plots of $\bar{p}^{Q}$ vs $T$ for SK systems of $L \times L \times L$ spins, for the values of $L$ shown, and $Q=1 / 4$ and $1 / 2$. Icons cover all error bars.

\section{NUMBER OF LINK MISMATCHES WHICH COME WITH LARGE SCALE EXCITATIONS}

In this section we give a definition of the fraction of mismatches, $\overline{\Delta f_{\mathrm{ml}}} Q$, it costs to create an excitation in the $-Q<q<Q$ range, where $0<Q<1$. The simplicity of the FB model is helpful in this respect. We introduce this nonrandom frustrated model in Sec. IV A. The definition of $\overline{\Delta f_{\mathrm{ml}}} Q$ as well as the results we obtain for the EA (and SK) model are given in Sec. IV B.

\section{A. The frustrated box model}

We define here a nearest-neighbor Ising model in which most bonds are ferromagnetic. For reasons given below, we term it the frustrated box (FB) model. Consider plane $\mathfrak{P}_{1}$, perpendicular to the $x$ axis, at $x=1 / 2$, which cuts all bonds between $x=0, y, z$, and $1, y, z$ sites. Similarly, $\mathfrak{P}_{2}$ at $x=L / 2+1 / 2$, cutting all bonds between $x=L / 2, y, z$ and $L / 2+1, y, z$. These planes divide the system into two equal portions. In this model, only nearest-neighbor spins interact. All bonds, except the ones that cut across $\mathfrak{P}_{1}$ and $\mathfrak{P}_{2}$, are of

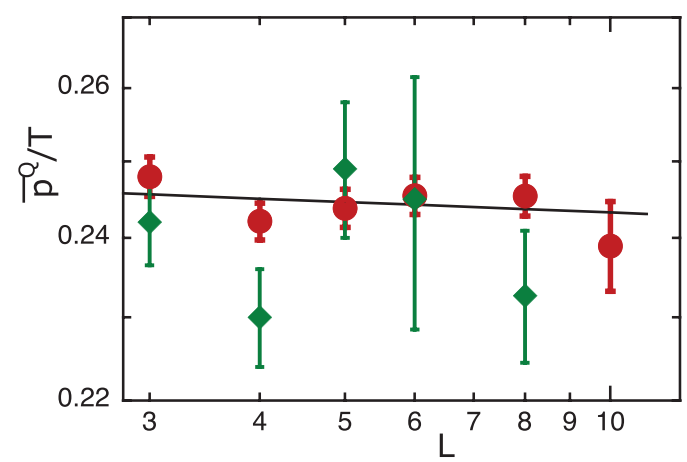

FIG. 4. (Color online) Log-log plots of $\bar{p}^{Q}$ vs $L$ for $Q=1 / 4$ of EA systems at $T=0.2$. All $\bullet$ stand for our own MC data and the straight line for the best $\bar{p}^{Q} \sim L^{-\theta}$ fit (given by $\theta \simeq 0.008$ ) to the data. The best fit gives $\chi^{2} \simeq 3.7$, and $\chi^{2} \simeq 10\left(\chi^{2} \simeq 8\right.$ ) follows from letting $\theta=0.04(-0.02)$. All stand for old data points from KPY for the same temperature and a slightly lower $Q=0.2$ value. [We have divided by two data values from KPY, because their reported values of $p(q)$ fulfill $\int_{0}^{1} d q p(q)=1$, instead of $\int_{-1}^{1} d q p(q)=1$.] 


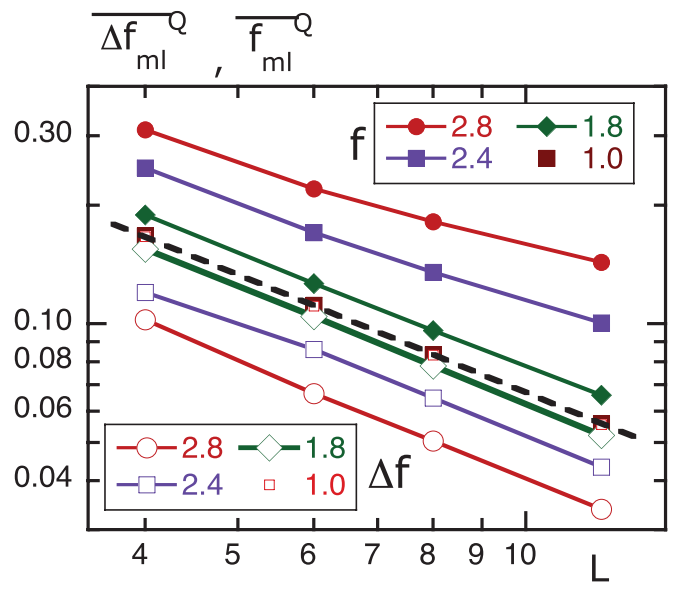

FIG. 5. (Color online) Plots of $\overline{\Delta f_{\mathrm{ml}}} Q$ and of $\overline{f_{\mathrm{ml}}} Q$, for $Q=1 / 2$, vs $L$ for FB systems at the $T$ values shown. The dotted line is for $2 / 3 L$. In the parallel tempered MC setup, $L=8 \mathrm{FB}$ systems were placed in thermal contact with heat baths equally spaced, by $\Delta T$, in the $6.5 \geqslant T \geqslant 0.8$ temperature range. For all $L \leqslant 8, \Delta T=0.02$, but $\Delta T=0.01$ for $L=12$.

strength 1 , that is, ferromagnetic. Half the bonds that cut across both $\mathfrak{P}_{1}$ and $\mathfrak{P}_{2}$ are of strength -1 , that is, antiferromagnetic, and the rest are of strength 1 . More precisely, all \pm 1 bonds that cut across both $\mathfrak{P}_{1}$ and $\mathfrak{P}_{2}$ are distributed on a checkerboard pattern. We apply periodic boundary conditions.

In the ground state, all spins within the box (that is, between planes $\mathfrak{P}_{1}$ and $\mathfrak{P}_{2}$ ) are parallel, and so are all spins outside the box. These two spin subsystems can point in the same or opposite directions. Thus, the ground state is (because of invariance under all-spin reversal) fourfold degenerate. The box defined by $\mathfrak{P}_{1}$ and $\mathfrak{P}_{2}$ and the system's boundary is the $3 \mathrm{D}$ analog of Toulouse's two-dimensional frustrated plaquettes. ${ }^{22}$ Hence, the "frustrated box" label.

The number of broken bonds in all ground states of the FB model is $L^{2}$, but the number of bond mismatches between two replicas is (in ground states) either 0 or $2 L^{2}$. Thus, $f_{\mathrm{ml}}=$ $1 / 3 L$ but $f_{\mathrm{ml}}(q=0)=2 / 3 L$. Plots of $\overline{f_{\mathrm{ml}}} Q$ vs $L$ are shown in Fig. 5 for $Q=1 / 2$ in FB systems at various temperatures. Note $\overline{f_{\mathrm{ml}}} Q=2 / 3 L$ at $T=1$, as expected for $T \ll T_{c} \simeq 4.5$.

Curves for $T=2.4$ and 2.8 in Fig. 5 clearly hint at a nonzero asymptotic value of $\overline{f_{\mathrm{ml}}} Q$. The right interpretation of this result comes easily for the FB model. Obviously, it isn't that $d_{s} \rightarrow 3$ as $L \rightarrow \infty$. Rather, bulk contributions to $\overline{f_{\mathrm{ml}}} Q$ compete with contributions (amounting to $2 / 3 L$ ) from the outer surfaces enveloping $q \sim 0$ excitations when $L$ is sufficiently large. Consider, for instance, $T \lesssim 2.5$. A $\exp (-12 / T)$ fraction of all spins point in the "wrong" direction then in large FB systems. This is the reason why $\overline{f_{\mathrm{ml}}} Q$ must cross over to a size-independent value [at $L \sim(1 / 18) \exp (12 / T)$ in the FB model]. Below, we subtract from $\overline{f_{\mathrm{ml}}} Q$ unwanted contributions.

\section{B. Number of link mismatches which come with large scale excitations in the EA model}

Let us first examine a simple picture of large and small scale excitations. In Fig. 6, the same cross section of an EA sample system of $10^{3}$ spins at $T=0.36$ is shown at four different times (consecutive times are at least $10^{6} \mathrm{MC}$ sweeps apart) of
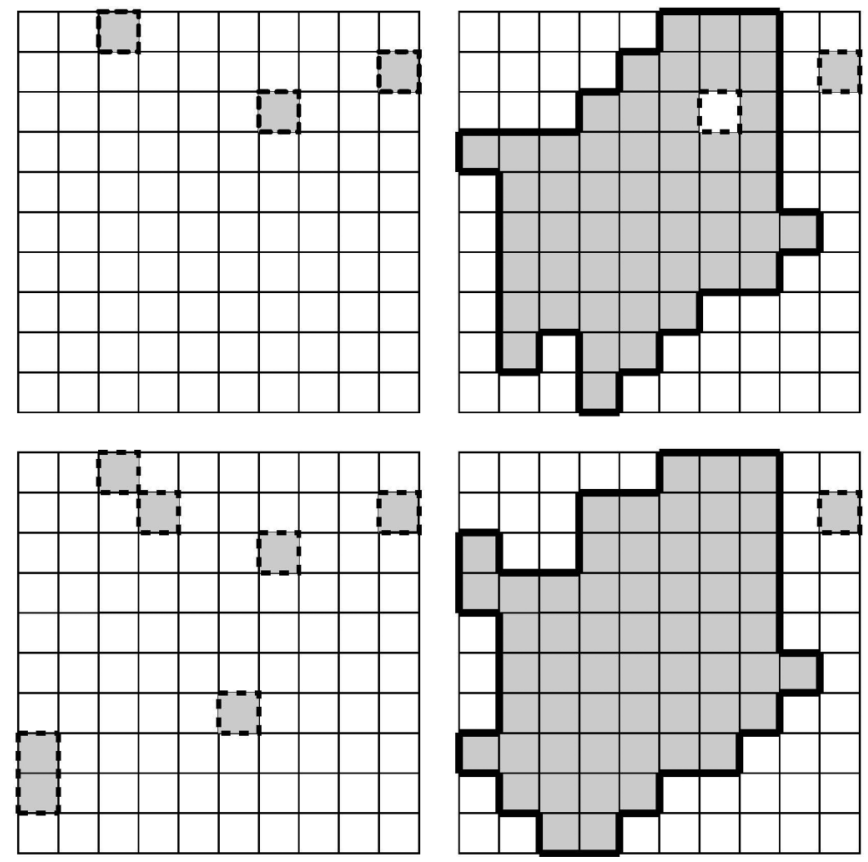

FIG. 6. Maps showing gray (white) squares for sites where $q_{i}=-1\left(q_{i}=+1\right)$ on a horizontal cut of a $10 \times 10 \times 10$ spin system. Outer surfaces of large scale excitations are shown with thick black lines. Broken lines stand for surfaces of small scale excitations. Different panels show maps observed at different times (consecutive times are at least $10^{6} \mathrm{MC}$ sweeps apart) from a simulation of an EA sample system at $T=0.36$. The spin-overlap parameter, taken over the whole system, had values $q \simeq 0.98,0.88,-0.06$, and -0.06 for the top left, bottom left, top right, and bottom right panels, respectively, at the times these images were recorded.

a single MC simulation. For a 3D picture of the outer surface of a large scale excitation in an EA system at $T=0.16$ see Fig. 7.

The general idea is to determine the area of the outer surface of large scale excitations, such as the ones on both right-hand panels of Fig. 6. We intend to do this by subtracting the total surface area of small scale excitations from the total (from small and large excitations) surface area.

For each system sample, we first obtain $f_{\mathcal{J} \text { ml }}(q)$ for each $q$ by adding $1 / 2\left(1-q_{l}\right)$ whenever $q$ is observed in a given MC run, and we finally divide the result by the number of times $q$ has been observed. Now, the average surface area over all excitations observed in a given sample whenever a value of $q$ is in the $-Q<q<Q$ range is given by

$$
\bar{f}_{\mathcal{J} \mathrm{ml}}^{Q} \equiv\left(1 / u_{\mathcal{J}}\right) \int_{-Q}^{Q} d q f_{\mathcal{J} \mathrm{ml}}(q) p_{\mathcal{J}}(q),
$$

where $u_{\mathcal{J}}=\int_{-Q}^{Q} d q p_{\mathcal{J}}(q)$, and by

$$
\bar{f}_{\mathcal{J} \mathrm{ml}}^{\emptyset} \equiv\left(1 / v_{\mathcal{J}}\right) \int_{|q|>Q} d q f_{\mathcal{J} \mathrm{ml}}(q) p_{\mathcal{J}}(q),
$$

where $v_{\mathcal{J}}=\int_{|q|>Q} d q p_{\mathcal{J}}(q)$, whenever $q$ is outside the $-Q<q<Q$ range. 


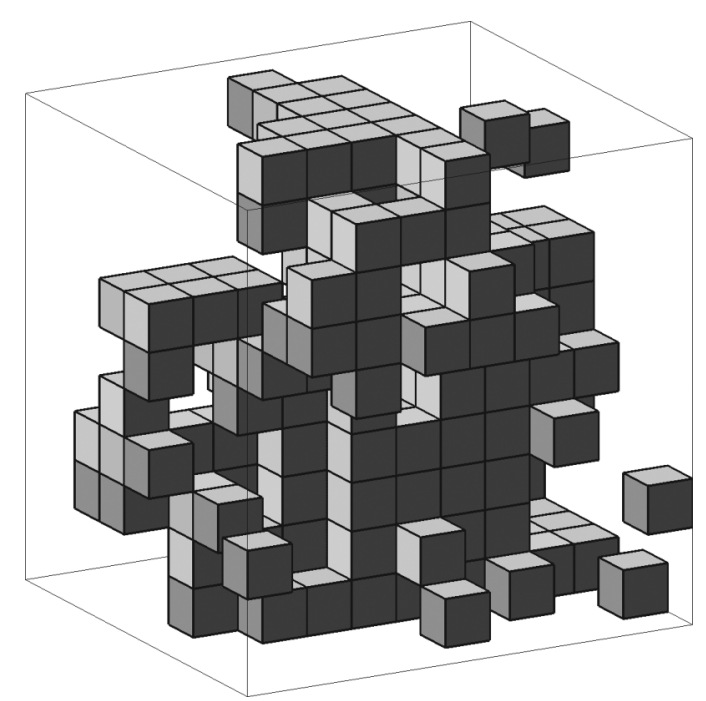

FIG. 7. Instantaneous 3D image showing outer surfaces on several small and one large scale excitation in an EA system sample at $T=0.16$. Flat surfaces on the framing cubic box are not excitation boundaries and should be disregarded as such. They arise from our inability to give an appropriate pictorial representation of periodic boundary conditions. At the time this image was recorded, $q \simeq 0.495$.

Finally, for the average FML it costs to create an excitation in the $-Q<q<Q$ range, we calculate

$$
\overline{\Delta f_{\mathrm{ml}}} Q \equiv\left\langle\overline{f_{\mathcal{J} \mathrm{ml}}} Q-\overline{f_{\mathcal{J} \mathrm{ml}}}\right\rangle_{\mathcal{J}}
$$

We have calculated $\langle\ldots\rangle_{\mathcal{J}}$ in the above equation by each of the following two procedures: (1) giving equal weight to all system samples for which $u_{\mathcal{J}}>0.1$ and (2) giving each sample a weight proportional to $\int_{-Q}^{Q} d q p_{\mathcal{J}}(q)$. Within statistical errors, we have obtained the same results from these two procedures.

Thus, $N_{l} \overline{\Delta f_{\mathrm{ml}}} Q$ is a reasonable definition of the outer surface area $\mathfrak{S}$ of an excitation with a $-Q<q<Q$ value. This definition excludes contributions from small scale excitations.

We can first check in Fig. 5 for the general behavior of $\overline{\Delta f_{\mathrm{ml}}} Q$ in the FB model. Data points approximately fall on straight lines. Furthermore, all are well fitted by $\overline{\Delta f_{\mathrm{ml}}} Q \sim$ $1 / L$, thus giving the desired value, $d_{s}=2$, for the dimension of planes $\mathfrak{P}_{1}$ and $\mathfrak{P}_{2}$, not only as $T \rightarrow 0$ but for nonzero temperatures as well.

Plots of $L^{0.39} f_{\mathrm{ml}}(q)$ vs $q$ are shown in Fig. 1 for EA systems of various sizes at $T=0.16$. However, departures from such scaling behavior can be observed, even over this limited range of system sizes, in analogous plots (not shown) for $T$ as small as $T \gtrsim 0.3$. This effect is more clearly exhibited in Fig. 8(a), where plots of $\overline{f_{\mathrm{ml}}} Q$ vs $L$ are shown for $Q=1 / 2$ and various temperatures.

A qualitatively different picture can be observed in Fig. 8(b). In it, plots of $\overline{\Delta f_{\mathrm{ml}}} Q$ vs $L$ are shown for the same $Q$ and $T$ as in Fig. 8(a). All data points shown for $\overline{\Delta f_{\mathrm{ml}}} Q$ in Fig. 8(b), which include temperatures up to $T \leqslant 0.8$, fall on straight lines. Consequently, $T \rightarrow 0$ extrapolations of their slope values is straightforward. From such extrapolations, we obtain $d-d_{s}=0.41$ (3). Within errors, this is in agreement with the value found in Refs. 9, 13, and 14 by a different method.

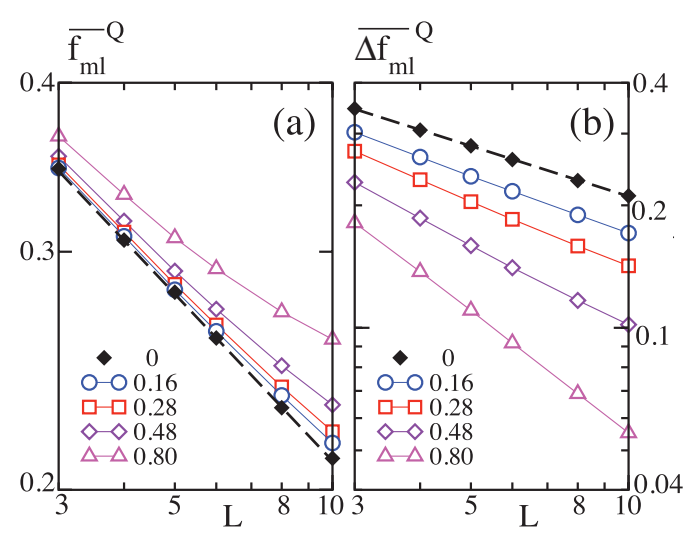

FIG. 8. (Color online) (a) Plots of $\overline{f_{\mathrm{ml}}} Q$ vs $L$, where $Q=1 / 2$, for EA systems of $L^{3}$ spins, for the values of $T$ shown. The data points $(\checkmark)$ shown for $T=0$ come from extrapolations such as the ones shown in Fig. 10. The slope of the dashed line going through the $T=0$ data points is 0.41 . (b) Same as in (a) but for $\overline{\Delta f_{\mathrm{ml}}} Q$. The $\checkmark$ data points are the same as in (a).

Incidentally, we note in Figs. 8(a) and 8(b) that whereas $\overline{f_{\mathrm{ml}}} Q$ decreases $\overline{\Delta f_{\mathrm{ml}}} Q$ increases as $T$ decreases. This is as expected, because, whereas the number of excitations (and thus $\overline{f_{\mathrm{ml}}} Q$ ) decreases as the temperature decreases, the cost $\left(\overline{\Delta f_{\mathrm{ml}}} Q\right)$ of creating an excitation increases as the temperature decreases (since higher temperatures imply a larger number of mismatched links to start with).

For $Q=1 / 4$, plots of $\overline{f_{\mathrm{ml}}} Q$ and $\overline{\Delta f_{\mathrm{ml}}} Q$ vs $L$ and various temperatures are shown in Figs. 9(a) and 9(b), respectively. Proceeding as above (for $Q=1 / 2$ ), we again arrive at $d-d_{s}=0.41(3)$. We thus infer this number to hold independently of $Q$ in the $Q \lesssim 1 / 2$ interval.

We can alternatively do a $T \rightarrow 0$ extrapolation of $\overline{\Delta f_{\mathrm{ml}}} Q$ and $\overline{f_{\mathrm{ml}}} Q$ for each value of $L$, as shown in Fig. 10 for $Q=1 / 4$ and $L=4$ and 10. Extrapolations from both curves meet, within errors, at the same point, as expected. The points thus obtained for $Q=1 / 2[Q=1 / 4]$ are plotted in Figs. 8(a) and 8(b) [Figs. 9(a) and 9(b)]. From log-log plots of the

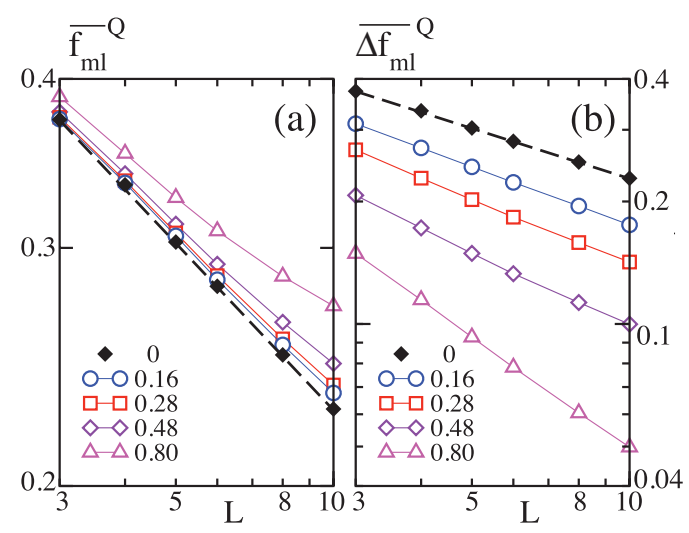

FIG. 9. (Color online) (a) Plots of $\overline{f_{\mathrm{ml}}} Q$ vs $L$, where $Q=1 / 4$, for EA systems of $L^{3}$ spins, for the values of $T$ shown. The data points $(\checkmark)$ shown for $T=0$ come from extrapolations such as the ones shown in Fig. 10. The slope of the dashed line going through the $T=0$ data points is 0.41 . (b) Same as in (a) but for $\overline{\Delta f_{\mathrm{ml}}} Q$. The $\checkmark$ data points are the same as in (a). 


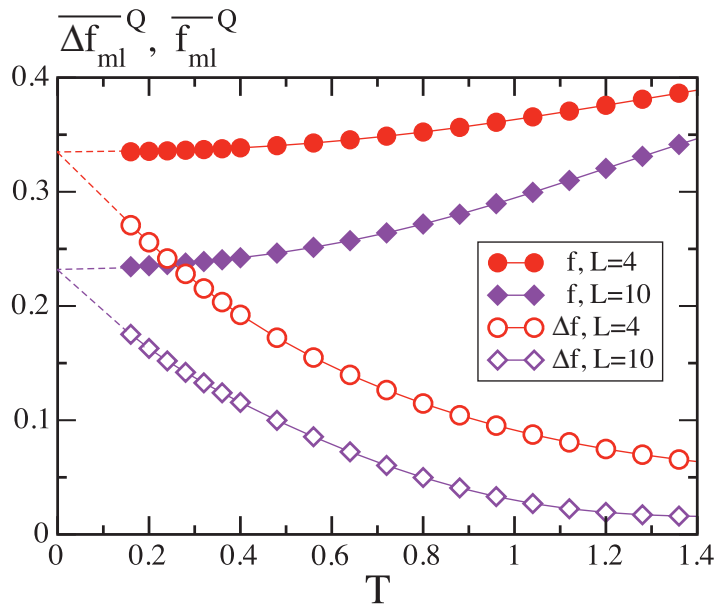

FIG. 10. (Color online) Plots of $\overline{\Delta f_{\mathrm{ml}}} Q$ and $\overline{f_{\mathrm{ml}}} Q$ vs $T$ for $Q=$ $1 / 4$ and the values of $L$ shown. The dotted lines are for our $T \rightarrow 0$ extrapolations.

zero-temperature curves thus obtained, we also obtained $d-d_{s}=0.41(3)$.

\section{CONCLUSIONS}

We have reported data for $p(q)$ from averages over large sets (numbers are shown in Table I) of EA and SK systems at very low temperature. The data for $\bar{p}^{Q}$ improves our confidence level in the conclusion that $p(q \sim 0)$ is nonzero and systemsize independent in the EA model. Future generation of more accurate data that would give $p(0) \sim 1 / L^{\theta}$ and $\theta>0.04$ in the $3 \leqslant L \leqslant 10$ range of $L$ values is rather unlikely. Thus, the conclusion KPY reached, ${ }^{9}$ that the EA model exhibits a clear trend away from the droplet scenario, is strengthened. Furthermore, our results are consistent with $p(0) / T \rightarrow 0.233(4)$ as $T \rightarrow 0$ in the EA model [and $p(0) / T \rightarrow 0.51(3)$ as $T \rightarrow 0$ in the SK model].

We have studied the fraction of link mismatches, $\overline{\Delta f_{\mathrm{ml}}} Q$, it costs to create an excitation with a $-Q<q<Q$ value. For a wide range of temperatures in the spin-glass phase, $\overline{\Delta f_{\mathrm{ml}}} Q$ seems to vanish, as in the TNT picture, ${ }^{12}$ in the macroscopic limit. Data points for $\overline{\Delta f_{\mathrm{ml}}} Q$ (for $Q=1 / 4$ and $1 / 2$ ) are consistent with $\overline{\Delta f_{\mathrm{ml}}} Q \sim 1 / L^{d-d_{s}}$ for all $T \lesssim T_{\mathrm{sg}}$. Furthermore, in agreement with results obtained in Refs. 9, 13, and 14 by a different method, we find $d-d_{s} \rightarrow 0.41(3)$ as $T \rightarrow 0$.

\section{ACKNOWLEDGMENTS}

We thank Centro de Supercomputación and Bioinformática and Laboratorio de Métodos Numéricos, both at Universidad de Málaga, for much computer time. Funding from the Ministerio de Economía y Competitividad of Spain, through Grant No. FIS2009-08451, is gratefully acknowledged.

\section{APPENDIX A: ERROR BARS}

We show here how statistical errors $\Delta\left(N_{s}\right)$ for $\bar{p}^{Q}$ depend on $N_{s}$, on system size, on $Q$, and on $T$ for $T \ll 1$.

Consider first the rms deviation $\delta p(q)$ of the probability density $p_{\mathcal{J}}(q)$ from its average over different samples. It is plotted vs $|q|$ in Fig. 3 of Ref. 1 for EA systems of various sizes at $T=0.1$. Because there is no self-averaging, $\delta p \gg p$, except near $q=1$. In addition, $\delta p(q) / p(q)$ does not decrease as $L$ increases. This has an unwanted implication, namely, that fractional statistical errors in $p(q)$ do not decrease as system size increases if $N_{s}$ remains constant.

To start, let $F_{\mathcal{J}}\left(q_{1}, q_{2}\right) \equiv p_{\mathcal{J}}\left(q_{1}\right) p_{\mathcal{J}}\left(q_{2}\right)$,

$$
G_{\mathcal{J}}(q \mid Q) \equiv \int_{0}^{Q} d q_{1} \int_{0}^{Q} d q_{2} \delta\left(q_{2}-q_{1}-q\right) F_{\mathcal{J}}\left(q_{1}, q_{2}\right)
$$

and let $G(q \mid Q)$ be the average of $G_{\mathcal{J}}(q \mid Q)$ over samples. We can then write that

$$
\Delta(1)^{2}=Q^{-2} \int_{-Q}^{Q} d q G(q \mid Q)-\left(\bar{p}^{Q}\right)^{2}
$$

follows. Now, let

$$
\int_{-Q}^{Q} d q G(q \mid Q) \equiv w G(0 \mid Q)
$$

define $w$. We also note that

$$
G(0 \mid Q)=Q\left[\left(\bar{p}^{Q}\right)^{2}+{\overline{(\delta p)^{2}}}^{Q}\right] .
$$

Here, the first term is much smaller than the second one for $T \ll 0.5$ and all $Q \lesssim 1 / 2$, in both the EA and SK models. This comes from the fact that, whereas $\bar{p}^{Q}$ vanishes as $T \rightarrow 0$, ${\overline{(\delta p)^{2}}}^{Q}$ does not. Therefore, $\Delta^{2}(1) \simeq(w / Q) \overline{(\delta p)^{2}} Q$ for $T \ll$ 0.5 and all $Q \lesssim 1 / 2$, whence

$$
\Delta\left(N_{s}\right) \simeq \overline{\delta p} Q \sqrt{\frac{w}{Q N_{s}}}
$$

follows immediately. This is clearly consistent with non-selfaveraging. It shows that $\Delta$ is, at least for the values of $L$ we study here, independent of $L$, for both the EA and SK models. Equation (A5) also shows how much precision is gained by averaging $p(q)$ over $-Q<q<Q$.

We can substitute into Eq. (A5) the low-temperature values of $w{\overline{(\delta p)^{2}}}^{Q} / \bar{p}^{Q}$ from Figs. 11(a) and 11(b) for the EA and SK models, respectively. Further substitutions of $\bar{p}^{Q}$ from Sec. III

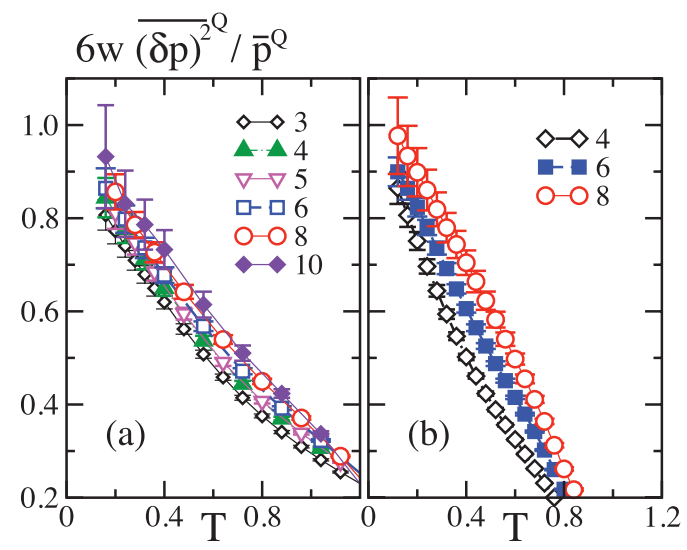

FIG. 11. (Color online) (a) Plots of $6 w{\overline{(\delta p)^{2}}}^{Q} / \bar{p}^{Q}$ vs $T$ for EA systems of $L$ linear sizes, as shown, and $Q=1 / 4$. (b) Same as in (a) but for the SK model. 
give

$$
\Delta\left(N_{s}\right) \simeq c \sqrt{\frac{T}{Q N_{s}}},
$$

where $c \simeq 0.2$ and 0.3 for the EA and SK models, respectively, at low temperatures. This is the desired expression.

\section{APPENDIX B: SWAP SUCCESS RATE AND EQUILIBRATION}

We show here (1) how we choose the success rate, $\alpha$, for state swapping (that is, for exchanging spin configurations) between two systems and (2) how we checked that equilibration was achieved in our simulations.

We first derive an expression for $\alpha$. In the parallel tempered MC algorithm, ${ }^{18,19}$ the probability, $P$ (ss $\mid \Delta E$ ), for state swapping to take place between systems 1 and 2 , at temperatures $T_{1}$ and $T_{2}$, where $\Delta E=E_{2}-E_{1}$, is given by $P(\operatorname{ss} \mid \Delta E)=1$ if $\Delta E \leqslant 0$ but $P(\operatorname{ss} \mid \Delta E)=\exp (-\Delta \beta \Delta E)$ if $\Delta E>0$. Now,

$$
\alpha=\int d \Delta E P(\text { ss } \mid \Delta E) P(\Delta E) .
$$

In thermal equilibrium, the probability that the energies of systems at $T_{1}$ and $T_{2}$ differ by $\Delta E$ is given by

$$
P(\Delta E) \propto \int d x e^{-x^{2} / 2 \sigma^{2}} e^{-(x-y)^{2} / 2 \sigma^{2}},
$$

where, neglecting variations in the specific heat (per spin) $c$ in the $T_{1}<T<T_{2}$ range, $N \sigma^{2}$ is the mean-square energy deviation coming from thermal fluctuations at both $T_{1}$ and $T_{2}$ :

$$
x=\frac{E-\langle E\rangle}{\sqrt{N}} \quad \text { and } \quad y=-\frac{\Delta E}{\sqrt{N}}+c \sqrt{N} \Delta T .
$$

It then follows that

$$
P(\Delta E)=(2 \sqrt{\pi N} \sigma)^{-1} e^{-y^{2} / 4 \sigma^{2}} .
$$

Substitution into Eq. (B1) yields, assuming $\Delta T \ll T_{1}<T_{2}$,

$$
\alpha \simeq \operatorname{erfc}(\Delta T \sqrt{c N} / 2 T)
$$

$\left[\operatorname{erfc}(\gamma)=\left(2 / \sqrt{\pi} \int_{\gamma}^{\infty} d x \exp \left(-x^{2}\right)\right]\right.$.

A choice of $\alpha \approx 0.5$ might seem to lead to efficient MC simulations, which, using Eq. (B5), would lead to $\Delta T \sqrt{c N} / 2 T \approx$ 0.48 . Note, however, that increasing $\Delta T$ does make $\alpha$ smaller, but it also implies that fewer random steps need be taken by a given state in order to travel from a system at the minimum temperature to one at the maximum temperature. Furthermore,

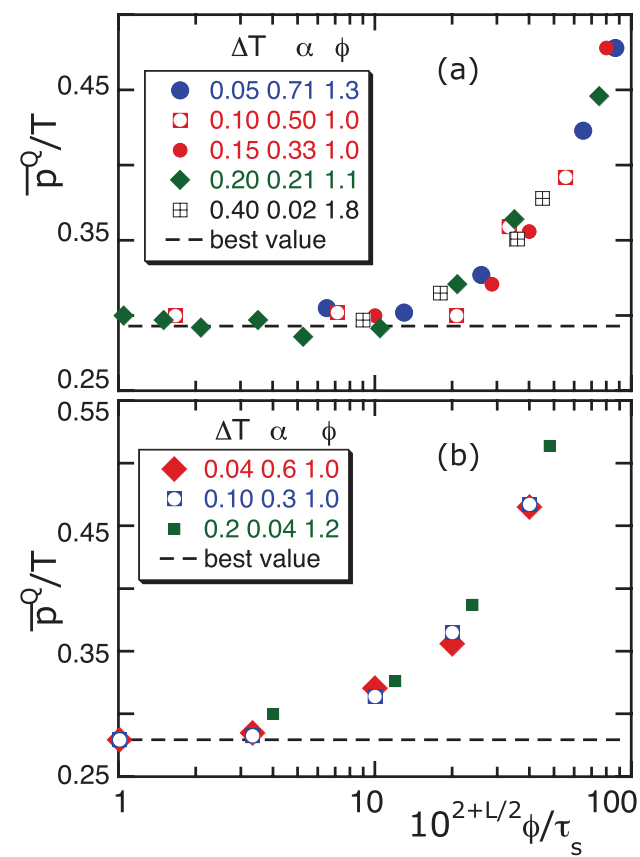

FIG. 12. (Color online) (a) Plots of the probability $\bar{p}^{Q} / T$ vs $10^{2+L / 2} \phi / \tau_{s}$ for $Q=1 / 2$ and at least $10^{4}$ sample EA systems of $6^{3}$ spins at $T=0.4$. $\phi$ is a scaling number which is chosen so all points fall as closely as possible to a single curve. The values of $\Delta T$, swap success rate $\alpha$ for swaps between systems at $T$ and $T+\Delta T$, and $\phi$ are as shown. The coldest system in the tempered MC setup from which these data points follow was at $T=0.4$. (b) Same as in (a) but for systems of $8^{3}$ spins at $T=0.2$, which were the coldest ones in the parallel tempered $\mathrm{MC}$ setup from which these data points follow.

smaller temperature differences imply fewer systems to be simulated, which leads to further computer time saving.

This point is illustrated in Fig. 12(a), where plots of $\bar{p}^{Q} / T$ vs $10^{5} \phi / \tau_{s}$ are shown for EA systems of $6 \times 6 \times 6$ spins at $T=0.4$. These data points come from tempered MC runs of sets of equally spaced temperatures. Values of the swap success rate, $\alpha$, between the two systems at the lowest pair of temperatures are given for each $\Delta T$, given by $T_{n+1}-T_{n}$, in Fig. 12(a). From the values of $\phi$ given in Fig. 12(a), we conclude that values of $\alpha$ as small as 0.2 do not lead to significantly slower simulations. Figure 12(b) is as Fig. 12(a) but for $L=8$ and $T_{\min }=0.1$, and $x$-axis values are for $10^{6} / \tau_{s}$. Note that even an $\alpha$ value as small as 0.04 only slows simulations down by $20 \%$.

\footnotetext{
*jefe@unizar.es

${ }^{1}$ J. F. Fernández and J. J. Alonso, Phys. Rev. B 86, 140402(R) (2012).

${ }^{2}$ S. F. Edwards and P. W. Anderson, J. Phys. F 5, 965 (1975).

${ }^{3}$ D. Sherrington and S. Kirkpatrick, Phys. Rev. Lett. 32, 1792 (1975).

${ }^{4}$ See Eq. (7) in M. Mézard, G. Parisi, N. Sourlas, G. Toulouse, and M. Virasoro, Phys. Rev. Lett. 52, 1156 (1984).

${ }^{5}$ M. Mézard, G. Parisi, and M. Virasoro, Spin Glass Theory and Beyond (World Scientific, Singapore, 2004).
}

${ }^{6}$ The number of spikes beyond some threshold height in large sets of system samples was found to increase in the SK model but remain approximately constant in the EA model as $L$ increases, in B. Yucesoy, H. G. Katzgraber, and J. Machta, Phys. Rev. Lett. 109, 177204 (2012).

${ }^{7}$ A. Billoire, L. A. Fernández, A. Maiorano, E. Marinari, V. Martín-Mayor, G. Parisi, F. Ricci-Tersenghi, J. J. Ruiz-Lorenzo, and D. Yllanes, arXiv:1211.0843 (2012). Note, however, that 
low-temperature behavior is inferred here from high-temperature data, where critical effects persist in fairly large EA systems in 3D.

${ }^{8}$ M. A. Moore, H. Bokil, and B. Drossel, Phys. Rev. Lett. 81, 4252 (1998).

${ }^{9}$ H. G. Katzgraber, M. Palassini, and A. P. Young, Phys. Rev. B 63, 184422 (2001).

${ }^{10}$ W. L. McMillan, J. Phys. C 17, 3179 (1984); D. S. Fisher and D. A. Huse, Phys. Rev. B 38, 386 (1988); M. A. Moore and A. J. Bray, J. Phys. C 18, L699 (1985); M. A. Moore, J. Phys. A 38, L783 (2005); see also A. A. Middleton, Phys. Rev. B 63, 060202 (2001).

${ }^{11}$ A note of caution on how deceptive finite-size behavior may sometimes be is given by A. A. Middleton, arXiv:1303.2253 (2013), where strong finite-size corrections in a toy droplet model are examined.

${ }^{12}$ F. Krzakala and O. C. Martin, Phys. Rev. Lett. 85, 3013 (2000).

${ }^{13}$ M. Palassini and A. P. Young, Phys. Rev. Lett. 85, 3017 (2000).

${ }^{14}$ T. Jörg and H. G. Katzgraber, Phys. Rev. Lett. 101, 197205 (2008).

${ }^{15}$ P. Contucci, C. Giardinà, C. Giberti, G. Parisi, and C. Vernia, Phys. Rev. Lett. 99, 057206 (2007); G. Parisi and F. Ricci-Tersenghi, Philos. Mag. 92, 341 (2012).

${ }^{16}$ Surface areas of large scale excitations in rather large systems (up to $L=32$ ) were also examined by R. Alvarez Baños, A. Cruz, L. A. Fernández, J. M. Gil-Narvion, A. Gordillo-Guerrero,
M. Guidetti, A. Maiorano, F. Mantovani, E. Marinari, V. MartínMayor, J. Monforte-García, A. Muñoz Sudupe, D. Navarro, G. Parisi, S. Pérez-Gaviro, J. J. Ruiz-Lorenzo, S. F. Schifano, B. Seoane, A. Tarancón, R. Tripiccione, and D. Yllanes, J. Stat. Mech. (2010) P06026. These simulations were carried out not too far below the critical temperature, and moving into the low-temperature regime by increasing the size of 3D EA systems at constant temperature is expected to require huge system sizes for the 3D EA model. Not surprisingly, it was concluded that the results obtained were "preasymptotic" (that is, far from the macroscopic regime).

${ }^{17}$ H. G. Katzgraber, M. Körner, and A. P. Young, Phys. Rev. B 73, 224432 (2006).

${ }^{18}$ K. Hukushima and K. Nemoto, J. Phys. Soc. Jpn. 65, 1604 (1996); see also J. F. Fernández, Phys. Rev. B 82, 144436 (2010); J. J. Alonso and J. F. Fernández, ibid. 81, 064408 (2010).

${ }^{19}$ For a critique of the parallel tempered MC method, see J. Machta, Phys. Rev. E 80, 056706 (2009).

${ }^{20}$ J. Vannimenus, G. Toulouse, and G. Parisi, J. Phys. (Paris) 42, 565 (1981).

${ }^{21}$ The slight downward displacement of KPY's data points with respect to our own can be accounted for by the slight difference between their $Q=0.20$ value and our own $Q=1 / 4$.

${ }^{22}$ G. Toulouse, Comm. Phys. 2, 115 (1977). 